\title{
Investigations of quench limits of the LHC superconducting magnets
}

\author{
Agnieszka Priebe, Bernd Dehning, Mariusz Sapinski, Minh Quang Tran, Arjan Verweij
}

\begin{abstract}
The NbTi-based Rutherford cables are used in the coils of the Large Hadron Collider (LHC) magnets. These cables are designed to operate with currents up to $13 \mathrm{kA}$ at temperatures 1.9 K. Beam losses can locally heat the superconducting cables above the critical temperature and cause a transition to the normal conducting state (quenching). The quench limit, i.e. the energy needed for this transition, is studied to determine maximum beam intensities and luminosity reach of the LHC. The amount of energy deposited in the coil cannot be measured directly. Therefore Geant4 simulations are used to correlate the deposited energy with the signal from secondary particles detected outside the magnet cryostat by ionization chambers. An orbital bump technique is used to induce controlled beam losses and provoke a quench. The energy deposition is analyzed in terms of various beam loss patterns and beam energies. The validation of the heat transfer code is presented. The development of the resistive zone is estimated and compared to the voltage measurements over the coils.
\end{abstract}

Index Terms - Accelerator magnets, Particle beams, Superconducting magnets

\section{INTRODUCTION}

$\mathrm{T}$ he design performance of the Large Hadron Collider (LHC) main magnets is based on the technology of the superconducting materials. The strands of applied cables are in a form of $\mathrm{Nb}$ - $\mathrm{Ti}$ filaments immersed inside a copper matrix. The superfluid helium bath provides cooling to $1.9 \mathrm{~K}$. Beam losses originating from the interactions with a residual gas, powering failures, orbit instabilities and collisions in the experiments can induce a transition from the superconducting state to the normal conducting state, i.e. quenching. The energy needed for an irreversible type of this process is called the minimum quench energy (MQE).

The LHC is equipped with two independent and complimentary systems that ensure safe operation of the

Manuscript received October 9, 2012.

A. Priebe is with the European Organization for Nuclear Research (CERN), CH - 1211 Geneva 23, Switzerland and École Polytechnique Fédérale de Lausanne (EPFL) (phone: +41 $22 \quad 76$ 75451; mail: Agnieszka.Priebe@cern.ch).

Bernd Dehning is with the European Organization for Nuclear Research $(\mathrm{CERN}), \quad \mathrm{CH}$ - 1211 Geneva 23, Switzerland (e-mail: Bernd.Dehning@cern.ch).

Mariusz Sapinski is with the European Organization for Nuclear Research (CERN), CH - 1211 Geneva 23, Switzerland (e-mail: Mariusz.Sapinski@cern.ch).

Minh Quang Tran is with École Polytechnique Fédérale de Lausanne (EPFL), Station 13, 1015 Lausanne, Switzerland (e-mail: minhquang.tran@epfl.ch).

Arjan Verweij is with the European Organization for Nuclear Research (CERN), $\mathrm{CH} \quad$ - 1211 Geneva 23, Switzerland (e-mail: Arjan.Verweij@cern.ch). accelerator in terms of protection against quenching. The Beam Loss Monitoring (BLM) system uses the ionization chambers for the secondary particle radiation dose measurements outside the magnet cryostat. Due to the applied thresholds, the beam is extracted from the machine before the quench occurs. In contrary, the Quench Protection System (QPS) reacts when a resistive volume is already developed. Typically, the voltage difference is measured between two parts of a superconducting coils. If the value exceeds $100 \mathrm{mV}$ within a time longer than $1 \mathrm{~ms}$, the beam is extracted from the accelerator to the beam dump. Furthermore, the quench heaters are triggered which results in an energy dissipation over an entire volume of the superconductor. The current is extracted from the coils through a bypass diode to a dump resistor.

The energy deposited inside the coil cannot be measured directly. Due to the magnet complexity and lack of space, no calorimeter could be installed in a coldmass. Therefore Geant 4 simulations are used to simulate it and correlate the results with the BLM signals. The reliability of this estimation is controlled by the BLM signal reproduction. These studies are essential for the determination of maximum beam intensities and luminosity reach of the LHC.

\section{Quench Test AT 3.5 TeV}

\section{Experimental conditions}

The experiment was conducted on $17^{\text {th }}$ October 2010 with the LHC $3.5 \mathrm{TeV}$ circulating proton beam. A technique of a three-corrector orbital bump was applied to induce controlled steady state losses. A corrector magnet (MCB) was deflecting the particles with an increasing magnetic field. This resulted in a rise of a beam impacting angle and the losses on the aperture in the region of the Main Quadrupole (MQ). The BLM thresholds were changed to avoid the beam dump before the quench occurred. After approximately $5.6 \mathrm{~s}$ the QPS system triggered the extraction of the beam from the accelerator and the firing of the quench heaters due to the voltage increase on the superconducting coils. During the test approximately 58\% of the initial bunch intensity $\left(1.85 \cdot 10^{10}\right)$ was lost in the accelerator. A rest of the particles were extracted to the beam dump. A propagation of the secondary particles shower was observed by six BLMs. Four monitors are located on the MQ cryostat and the other two on the Main Dipoles (MB). Experimental data analysis was presented in [1].

\section{Beam size}

The beam emittance $\varepsilon$ is constant along the accelerator and can be calculated from the following equation: 


$$
\varepsilon=\frac{\sigma^{2}}{\beta}
$$

where $\sigma$ denotes a beam size and $\beta$ stands for the $\beta$-function. The beam size was measured with the Wire Scanner, $\sigma_{W S}$,vertical $=876 \mu \mathrm{m}$. Since $\beta_{W S}=404.55 \mathrm{~m} \mathrm{[2]}$, the beam emittance at the beginning of the test was $\varepsilon=1.9 \cdot 10^{-3} \mu \mathrm{m}$. The emittance blow up is not expected during the experiment.

\section{GEANT4 SIMULATIONS}

The Geant4 simulations concern the LHC arc half cell. The geometry representation includes the MQ with a sextuple (MS) and the MCB, a downstream MB and the interconnection between the MB and the MQ. Instead of six fixed BLMs outside the magnet cryostat, two long pseudodetectors were simulated to investigate a propagation of the secondary particles along the machine. Beam 2 was moving anti-clockwise and hitting the aperture with an impacting angle of $202 \mu \mathrm{rad}$ in the vertical plane. This value was calculated from the orbit bump settings. The exact loss pattern along the MQ vicinity is unknown. Therefore 71 loss scenarios were simulated (Fig.1) with a longitudinal spacing of $0.1 \mathrm{~m}$ which was chosen to be less than a nuclear interaction length $(0.15 \mathrm{~m}$, [3]). A combination of the weighted results allows imposing any considered beam loss pattern without repeating the long simulation process. In the first approach, it was assumed that the incoming particle bunch had a Gaussian shape which remained unchanged by the bump. Moreover the loss should also posses the same properties as the travelling beam, i.e. a projection of the beam should have a normal distribution.

The energy deposited inside the superconducting coils was estimated with the resolution of the implemented cylindrical bin size: $\Delta r=5.13 \mathrm{~mm}, \Delta \varphi=4^{\circ}$ and $\Delta z=9.83 \mathrm{~mm}$.

The simulation algorithm for an estimation of the BLM signal combines the number of secondary particles, their type and energy with the response functions for nine angles of incoming particles and was described in [1].

There are several hypotheses on the loss patterns. Due to the experimental conditions, two loss scenarios were studied. In the first case it was assumed that the maximum of the Gaussian distribution occurs in the centre of the Main Quadrupole (the mean $\mu=0 \mathrm{~m}$ ) since the $\beta$-function at this position (focusing quadrupole) reaches its local maximum. Moreover, the bump amplitude has also its maximum in the MQ. The other loss location was taken to be in the corrector magnet upstream of the MQ $(\mu=2.5 \mathrm{~m})$.

In this paper an average of beam shapes over a time of $5.6 \mathrm{~s}$ is studied. No time-dependent changes of the emittance and the loss pattern are taken into account. The Geant 4 simulations are compared to the integrated losses over $6 \mathrm{~s}$.

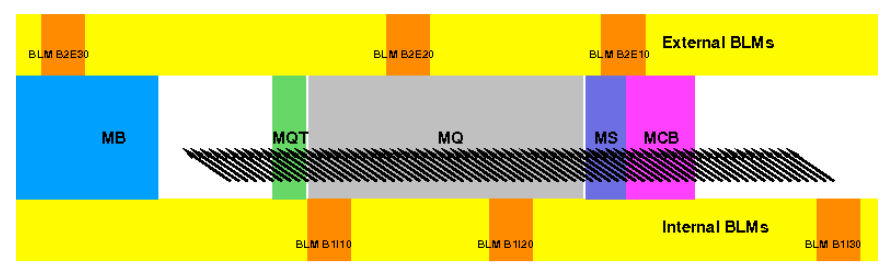

Fig. 1. The LHC magnet representation. 71 point-like losses were simulated along the vicinity of the MQ.

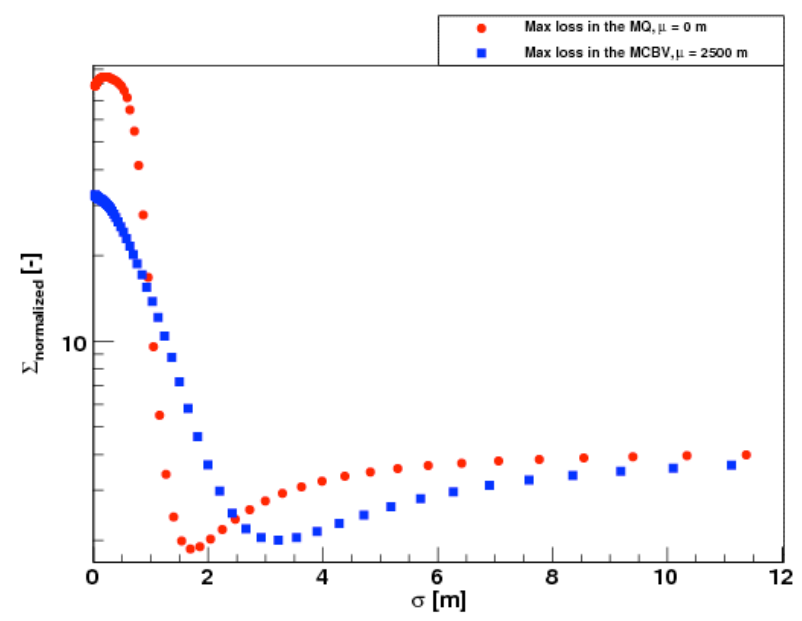

Fig. 2. The most probable loss pattern is determined by a minimum of the error coefficient $\Sigma_{\sigma}$,normalized (logarithmic scale).

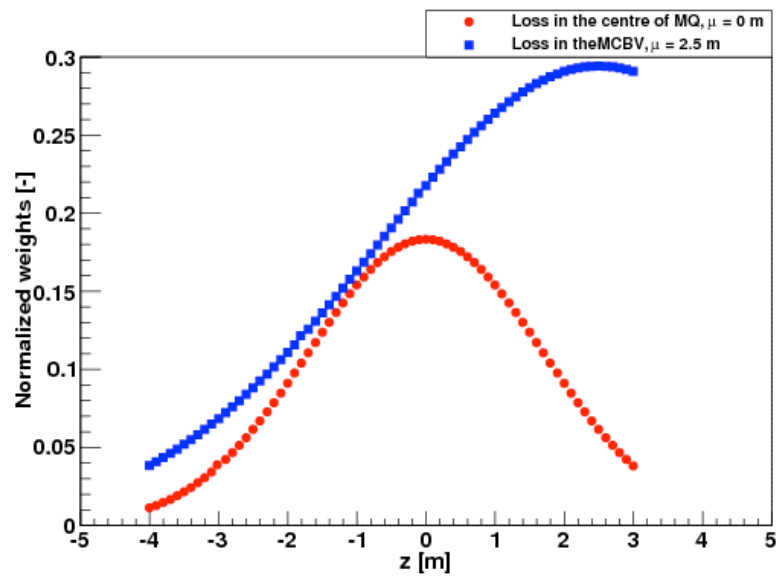

Fig. 3. Estimated loss patterns.

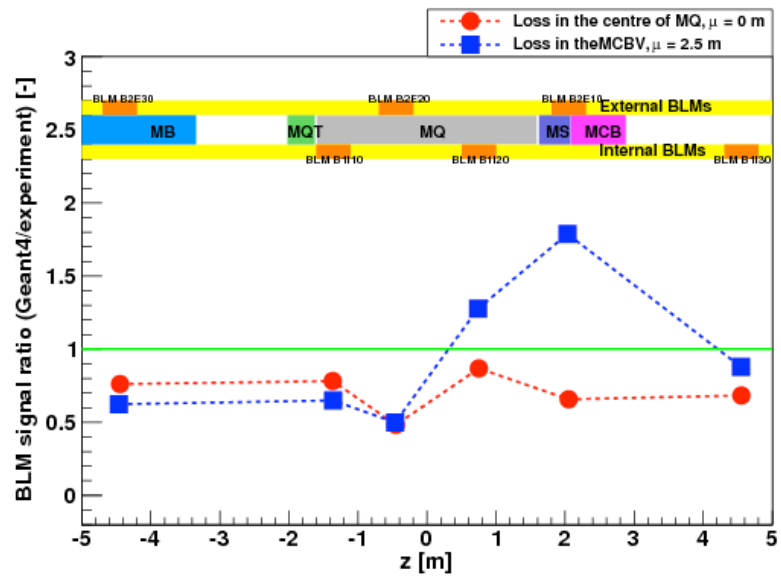

Fig. 4. Ratios between simulated and measured BLM signals. 
TABLE I

INITIAL BEAM SIZE AT THE LOCATION OF MQ AND MCB.

\begin{tabular}{ccccc}
\hline \hline Location & $\mu[\mathrm{m}]$ & $\beta[\mathrm{m}]$ & $\sigma_{\text {beam }}[\mu \mathrm{m}]$ & $\sigma_{\text {loss }}[\mathrm{m}]$ \\
\hline MQ centre & 0 & 184 & 591 & 2.92 \\
$M C B$ & 2.5 & 176 & 577 & 2.86 \\
\hline
\end{tabular}

It is assumed that the initial loss sizes can be simply calculated using a transformation of Equation (1). The beam loss is a projection of the beam on the beam screen surface if no external forces are involved. The results are given in Tab.1. Nevertheless, a strong magnetic field of the MQ was focusing the particles in the vertical plane. Thus, the normal distribution should be conserved but an average beam width $\sigma$ is unknown. The Gaussian $\sigma$ was investigated as a free parameter and compared to the experimental signals of the BLMs to find the most likely scenario. This was determined by an error parameter $\Sigma_{\sigma, \text { normalized }}$ defined as

$$
\Sigma_{\sigma, \text { normalized }}=\sum_{i=1}^{6}\left(\frac{l_{\sigma, i}^{s i m}-l_{i}^{\text {exp }}}{l_{\sigma, i}^{s i m}}\right)^{2}
$$

Variables $l_{\sigma, s i m}$ stands for the simulated signals and $l_{\text {exp }}$ denotes the experimental data for the given values of $\mu$ and $\sigma$. A contribution of all six BLMs is included in the iteration over $i$.

The optimal beam sizes, i.e. characterizing the mimimum of $\Sigma_{\sigma, \text { normalized, }}$, were found (Fig.2) to be $\sigma_{M Q}=1.7 \mathrm{~m}$ and $\sigma_{M C B}=$ $3.2 \mathrm{~m}$ for the maximum losses in the MQ and the MCB, respectively. A comparison with the initial conditions indicates that it is more probable that the beam loss occurred in the MQ centre since the beam size decreases under the influence of the focusing force.

The applied loss shapes are given on Fig. 3. The ratios of the simulated and measured BLM signals are presented on the Fig. 4. The results of the losses in the MQ centre are underestimated. Nevertheless they show a good agreement with the experimental data.

The normalized energy deposition density inside the MQ superconducting coils and the number of secondary particles reaching the BLMs are presented for both cases in the longitudinal direction (Fig. 5 and Fig. 6, the beam travels from the right to the left). The spread of $E_{\text {dep }}$ inside the coil is strongly affected by the loss location and reaches maximum of around $0.27 \cdot 10^{-6} \mathrm{~mJ} / \mathrm{cm}^{3}$ per proton in the case of the loss in the MQ. Independently on the loss scenario, the maximum number of particles is detected in the interconnection region between the MB and the MQ. Therefore the additional monitors will be installed in these locations for the future tests to increase the resolution in a loss detection. The head of the distribution is greater for losses localized in the quadrupole magnet.

Fig. 7 presents the radial energy density distribution in the most impacted position of the coil. The fit function depends on the coil radius $r$ and three fit parameters $p_{0}, p_{1}$, and $p_{2}$ :

$$
E_{d e p}=p_{0}\left(r-p_{1}\right)^{p_{2}}
$$

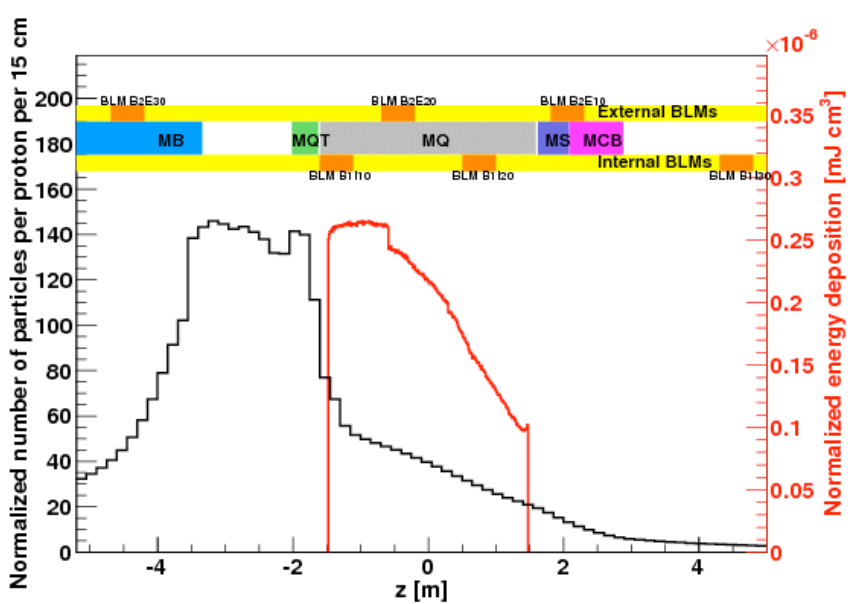

Fig. 5. Geant4 simulations: loss location in the center of the quadrupole (MQ).

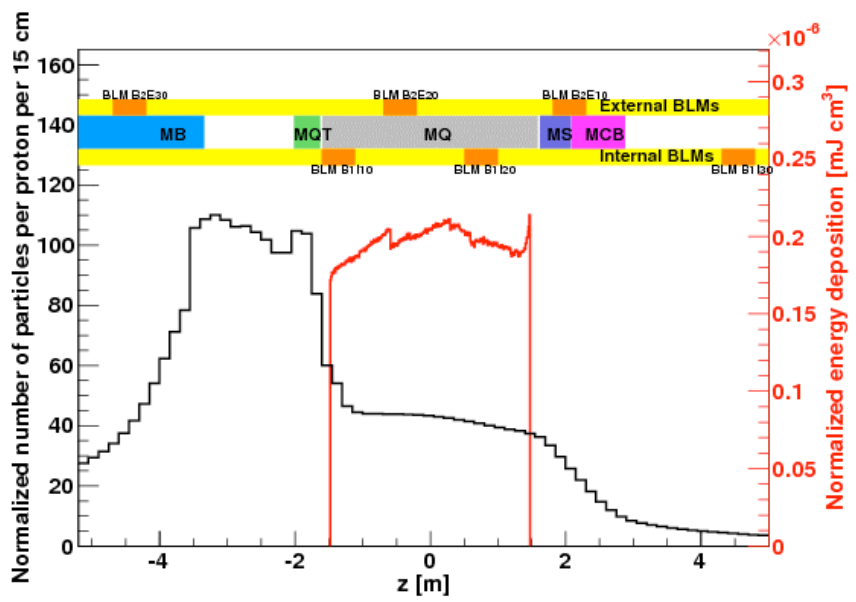

Fig. 6. Geant4 simulations: loss location in the center of the corrector magnet (MCB).

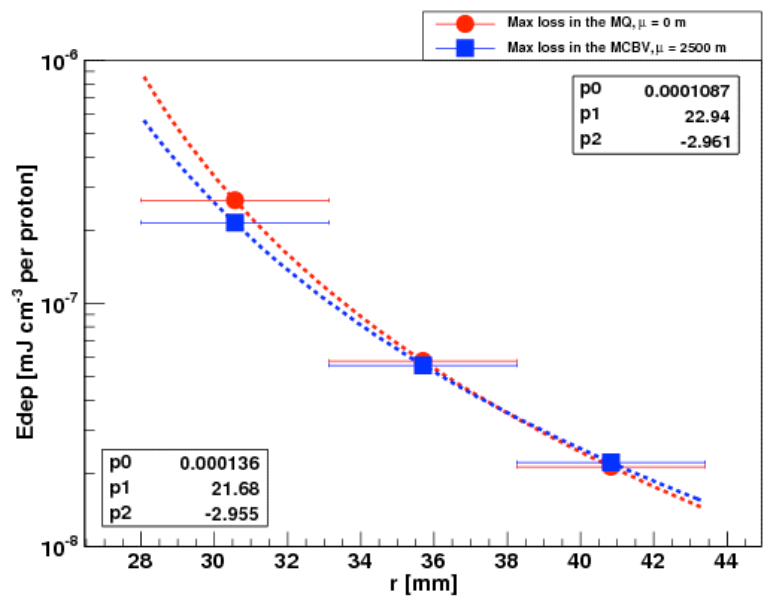

Fig. 7. Radial energy density distribution inside the MQ superconducting coil (inner layer).

These functions were used as an input to the QP3 heat transfer code [4] to determine the quench limits.

\section{QUENCH LIMIT}

The energy level at which a quench of the superconducting cables occurs can be calculated with the QP3 heat transfer 
TABLE II

LENGTH OF THE QUENCHED LHC MAGNET

\begin{tabular}{ccc}
\hline \hline RRR [-] & $\rho_{\mathrm{Cu}}[\Omega \mathrm{m}]$ & $1[\mathrm{~m}]$ \\
\hline 150 & $2.25 \cdot 10^{-10}$ & 1.01 \\
200 & $1.96 \cdot 10^{-10}$ & 1.23 \\
\hline
\end{tabular}

code. The loss distribution in time, the radial energy distribution inside the superconducting coil, the magnet current (5.6 kA for $4 \mathrm{TeV}$, which means $156 \mathrm{~A}$ per a strand) and loss duration are the main inputs to the program. The average energy $E_{\text {avg }}$ is the most important output.

The consideration of the longitudinal loss shape does not change the quench level value estimated with the QP3 code when comparing to the point-like loss considerations [1] $\left(0.55 \mathrm{~J} / \mathrm{cm}^{3}\right)$. The results based on the Geant 4 simulations are fitted better by a factor of $\approx 4$ giving values of $1.49 \mathrm{~J} / \mathrm{cm}^{3}$ in case of the losses inside the MQ and $1.19 \mathrm{~J} / \mathrm{cm}^{3}$ for losses in the corrector magnet. Only a small part of the difference between these two methods can be explained by the fact that the energy given by the QP3 code has a meaning of the MQE. The Geant4 estimations provide the quench limit after more than $100 \mathrm{~ms}$ due to the QPS system acceptance margin.

\section{RESISTIVE VOLUME}

The QPS system detects the voltage difference between two halves of one quadrupole, each having an inductance $L$ of $2.8 \mathrm{mH}$. The threshold of the QPS is set at $100 \mathrm{mV}$ with an evaluation time of $10 \mathrm{~ms}$. Once the threshold is reached, the quench heaters are fired within a few ms. Then, there is a 20 $200 \mathrm{~ms}$ delay between the moment when the quench heaters are triggered and the moment when they effectively heat up the coils above the critical temperature of the superconductor.

The resistivity of the copper matrix of the LHC cables at temperatures up to about $20 \mathrm{~K}$ can be approximated by:

$$
\rho_{C u}=1.7 \cdot 10^{-8} \frac{1}{R R R}+0.4 \cdot 10^{-10} B
$$

with $R R R$, the residual resistivity ratio of the LHC strands [5] which depends on the copper quality, heat treatment and annealing. The RRR of the LHC SC coils is usually greater than 150 [6].

The QPS voltage measurements are shown in Fig. 7. $U_{-}{ }_{-} E X T$ and $U_{-} 2_{-} E X T$ are the voltages on the two halves of the magnet, $U_{-} H D S_{-} 1$ is the voltage on the quench heaters, and $U \_Q S O \_E X T$ is the sum of $U_{-} 1 \_E X T$ and $U_{-} 2_{-} E X T$.

The resistance $R$ is defined as

$$
R=\frac{U}{I}=\rho \frac{l}{S}
$$

with $l$ - the cable length and $S$ - the copper cross-section of the cable. The voltages include an inductive component, so the energy dissipation is given by

$$
E_{\text {dis }}=I \cdot\left(U_{-} 1_{-} E X T-L \frac{d I}{d t}\right) \cdot t
$$

with $I$ - the current in the magnet. $U_{-} Q S O \_E X T$ increased from 0 to $100 \mathrm{mV}$ within about $60 \mathrm{~ms}$ which resulted in triggering the quench heaters and energy extraction (EE) with a time

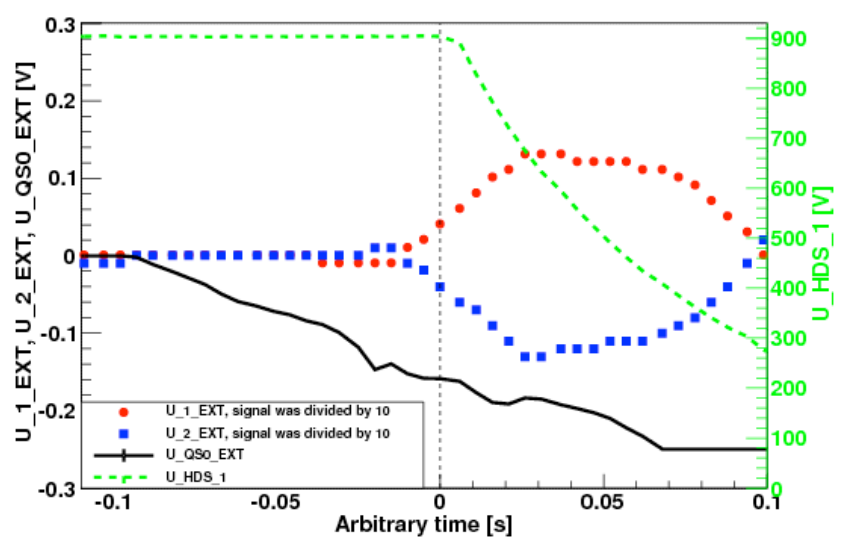

Fig. 7. QPS measurements. Time axis is given with respect to the quench detection at $U_{-} Q S O_{-} E X T=100 \mathrm{mV}$.

constant $\tau \approx 9.2 \mathrm{~s}$. Thus, the initial time derivative of current is about $-600 \mathrm{~A} / \mathrm{s}$ causing an inductive voltage on $U_{-}{ }_{-} E X T$ of $1.7 \mathrm{~V}$. Due to the resolution, signal filtering and the large inductive component, the resistive part cannot be accurately distinguished. During the Quench Test the beam was targeted on one magnet pole only so it can be assumed that $U \_Q S O \_E X T=U_{-}{ }_{-} E X T$ or $U_{-} Q S O \_E X T=U_{-}{ }_{-} E X T$. Knowing that up to $U_{-} Q S O \_E X T=100 \mathrm{mV}$ the voltage is purely resistive, the normal length of the cable can be estimated using $S \approx 13 \cdot 10^{-6} \mathrm{~m}^{2}, B=2.8$ in the most exposed part of the cable and $I=5.4 \mathrm{kA}$. Table II contains results of calculations for different values of $R R R$. During the experiment, the quenching occurred on the cable length of $1.01-1.23 \mathrm{~m}$. The Geant4 data analysis is ongoing.

\section{OUTLOOK}

The loss pattern determination is the next step of the performed analysis. Both, the beam loss location and the loss size will be optimized. Moreover an asymmetrical shape of the incoming beam will be implemented. The time evolution of the losses is foreseen. The SixTrack simulations are expected to provide more information in the loss pattern studies.

A new version of the Geant4 magnet representation was built and is being simulated.

\section{CONCLUSION}

The studies on the LHC quench limits and the loss propagation along the machine are required for setting the safe thresholds on the BLM monitors.

The three-corrector orbital bump technique was used for the steady state losses induction. The performed Geant4 simulations were compared to the measured observables. A consideration of the beam shape improved the agreement between Geant4 data and QP3 results by a factor of 4 with respect to the point-like losses. The simulations indicate that the loss location is more likely to be in the centre of the Main Quadrupole. Moreover the secondary particles distribution indicates the locations where additional monitors should be installed for the future Quench Test to improve the loss detection resolution. 


\section{REFERENCES}

[1] A. Priebe et al., "Beam-induced quench test of a LHC Main Quadrupole", Proceedings of IPAC2011 (WEPC172), San Sebastián, Spain.

[2] Jmad, http://wikis.cern.ch/display/JMAD/Download (program available online)

[3] J. B. Jeanneret, D. Leroy, L. R. Oberli, T, Trenkler, " Quench levels and transient beam losses in LHC magnets", CERN-LHC-Project-Report-44, Geneve, 1996.

[4] A. Verweij, "QP3 Users Manual”, CERN/EDMS 1150045

[5] A. Apollonio et al., "Measurement of the Residual Resistivity Ratio of the busbars copper stabilizer of the 13kA circuits of the LHC", CERNATS-Note-2012-032 TECH, CERN, Geneve, Switzerland

[6] The LHC Design Report, Volume I, Chapter 7: "Main Magnets in the Arcs", http://hc.web.cern.ch/lhc/lhc-designreport.html

[7] A. Priebe et al., "ADT fast losses MD", to be published

[8] ROXIE program, courtesy of Bernhard Auchmann, CERN, Geneve, Switzerland 